\section{Efectos del Programa “Ejercicio Dirigido-Paseos Saludables en Entornos Curativos Exteriores" en ancianos hospitalizados por trastornos depresivos}

\section{Effects of the Program "Directed Exercise-Healthy Walks in Healing Outdoor Environments" in elderly hospitalized for depressive disorders}

Raquel Sáenz Mendía, ,*

María Inés Gabari Gambarte ${ }^{2}$

José María García García ${ }^{3}$

Marta Ferraz Torres ${ }^{4}$

Rosana de la Rosa Eduardo ${ }^{5}$

Moad Al-Rahamneh ${ }^{6}$

1. Enfermera. Doctora en Ciencias de la Salud. Universidad Pública de Navarra. Facultad de Ciencias de la Salud. Pamplona. Navarra. España.

2. Doctora en Psicología y Pedagogía. Universidad Pública de Navarra. Grupo de Investigación Educación y Salud-EyS. Pamplona. Navarra. España.

3. Enfermero. Ldo. en Antropología Social y Cultural. Universidad Pública de Navarra. Facultad de Ciencias de la Salud. Pamplona. Navarra. España.

4. Enfermera. Doctora en Ciencias de la Salud. Universidad Pública de Navarra. Facultad de Ciencias de la Salud. Pamplona. Navarra. España.

5. Enfermera. Doctora en Salud Pública. Universidad Pública de Navarra. Facultad de Ciencias de la Salud. Pamplona. Navarra. España.

6. Doctor en Salud Pública. The Eastern Mediterranean Public Health Network. Jordan.

${ }^{*}$ Autor para correspondencia.

Correo electrónico: raquel.saenz@unavarra.es (Raquel Sáenz Mendía).

Recibido el 14 de octubre de 2019; aceptado el 28 de diciembre de 2019

\section{RESUMEN}

Objetivo: Analizar los efectos de un programa integrado de "Ejercicio Dirigido-Paseos Saludables en Entornos Curativos Exteriores" (ED + PSECE) en el bienestar físico y psicosocial de pacientes con trastorno depresivo, mayores de 65 años, ingresados en la Unidad de Agudos de Hospitalización Psiquiátrica. Método: Estudio piloto experimental con un grupo intervención (GI, $\mathrm{n}=32$ sujetos) y un grupo control (GC, $\mathrm{n}=32$ sujetos). Las mediciones pre- y postintervención se realizan con el índice de Barthel, el test de Guralnik Short Physical Performance Battery (SPPB), la escala de Hamilton, el Get Up and Go Test y la taxonomía NOC. Resultados: Se encuentran mejoras significativas del GI en funcionalidad según Barthel $(0,036)$ y en posición corporal según NOC $(0,025)$, así como disminución de síntomas depresivos según Hamilton (0,001). Conclusión: La actividad física contribuye a la reducción de síntomas negativos de los trastornos depresivos y a la mejora de la funcionalidad para el desempeño de actividades básicas de una vida autónoma, siendo fundamental el rol desempeñado por el profesional de enfermería para la adherencia al programa de los pacientes ancianos hospitalizados en una unidad de salud mental.

PALABRAS CLAVE: Salud del anciano, depresión, terapia por ejercicio, autonomía personal, enfermería psiquiátrica.

\section{ABSTRACT}

Objective: To analyze the effects of an integrated "Healthy ExerciseWalks in Outdoor Healing Environments" on the physical and psychosocial well-being of patients with depressive disorders over 65 years of age admitted to acute unit of Psychiatric hospitalization. Method: Experimental pilot study with an intervention group ( $\mathrm{N} \mathrm{IG=32} \mathrm{subjects)} \mathrm{and} \mathrm{a} \mathrm{control} \mathrm{group} \mathrm{(} \mathrm{N} \mathrm{CG}=32$ subjects). Pre- and post-intervention measurements were made with the Barthel index test, Guralnik Short Physical Performance Battery (SPPB), Hamilton scale, a Get Up and Go Test and the Taxonomy NOC. Results: Significant progress of the IG is in functionality as Barthel test $(0.036)$ and in corporal position as NOC (0.025), as well as decrease of depressive symptoms according to Hamilton (0.001). Conclusion: Physical activity contributes to the reduction of negative symptoms of depressive disorders and to the improvement of functionality for the performance of basic activities of an autonomous life, the role played by the professional nurse for adherence to the program of elderly patients hospitalized in a mental health unit.

KEYWORDS: Health of the elderly, depression, exercise therapy, personal Autonomy, psychiatric nursing.

\section{- INTRODUCCIÓN}

La depresión es una de las patologías psiquiátricas más frecuentes e incapacitantes en el colectivo de población geriátrica y, según estimaciones de la Organización Mundial de la Salud (OMS) ${ }^{1}$, en el año 2030 se convertirá en la primera causa de discapacidad en el mundo. Este trastorno engloba una amplia gama de problemas de salud mental caracterizados por la ausencia de afectividad positiva (una pérdida de interés o de la capacidad de disfrutar en actividades que normalmente eran placente- ras), bajo estado de ánimo y una serie de síntomas emocionales, físicos, conductuales y cognitivos asociados ${ }^{2}$. Estas manifestaciones pueden aparecer a cualquier edad; sin embargo, la forma de presentación puede diferir en personas mayores ${ }^{3}$.

En los períodos de hospitalización, los síntomas negativos y de inhibición en personas ancianas pueden agudizarse al encontrarse en un entorno hostil y desconocido. Se limitan sus actividades de ocio y tiempo libre, lo que conlleva una disminución de la actividad física y la permanencia en la cama como un refugio para escapar de la realidad. Esto 
supone pérdida de la capacidad funcional, mayor riesgo de comorbilidades junto con agudización de los síntomas vinculados a la depresión, reforzados por la inactividad física ${ }^{4-6}$.

Numerosas investigaciones constatan que los trastornos depresivos ${ }^{7,8}$ precisan de un tratamiento combinado para la integración de los principios farmacológicos y psicoterapéuticos. En los últimos años, estos dos pilares se han transformado en tres, con la suma de la prescripción de ejercicio físico como tratamiento importante para la mejora de la forma física, el alivio de los síntomas de la enfermedad y la sensación de bienestar emocional? .

Los estudios recientes sobre la relación entre la actividad física y la depresión en ancianos ${ }^{10,11}$ encuentran que existe una influencia positiva del ejercicio físico en la reducción de los síntomas depresivos y en la mejoría de la aptitud funcional, lo que sugiere una relación inversa entre esas variables. Según el plan de acción integral de la $\mathrm{OMS}^{12}$ sobre la salud mental para el período 2013-2020, se incluye la evaluación de los efectos de los programas de ejercicio físico en los síntomas depresivos, calidad de vida y autoestima en personas de edad avanzada.

Uno de los apartados fundamentales de estos programas se centra en la mejora de la condición física para la prevención de caídas en ancianos. Determinadas alteraciones del estado de salud, como la inhibición psicomotora, la comorbilidad, los problemas de audición y visión, la polifarmacia, la privación de sueño junto con los fármacos utilizados para el tratamiento del síndrome depresivo son factores que incrementan el riesgo de caídas en estos pacientes ${ }^{13,14}$.

Durante el proceso de hospitalización psiquiátrica, los profesionales de enfermería desempeñan un rol facilitador esencial. El acompañamiento, la supervisión y la realización de programas de ejercicio físico individual y sistemático, especialmente adaptado a las necesidades de estos ancianos, contribuyen a la mejora, por una lado, del estado de ánimo, minimizando los síntomas negativos y, por otro, de la actividad motriz, en relación con la flexibilidad, la fuerza, el equilibrio y la coordinación ${ }^{15}$.

El objetivo del estudio piloto es analizar los efectos en el bienestar físico y psicosocial de un programa integrado de "ejercicio dirigido-paseos saludables en entornos curativos exteriores" (ED + PSECE) en pacientes con trastorno depresivo, mayores de 65 ańos, ingresados en una unidad de agudos de hospitalización psiquiátrica. Los objetivos secundarios son: identificar el grado de disminución de inhibición psicomotora y trastornos depresivos, así como la mejora en funcionalidad en el desempeńo de actividades de la vida diaria.

\section{- MÉTODO}

\section{Diseño}

Estudio experimental prospectivo con un grupo de intervención (GI) que realiza el programa de ejercicios (ED + PSECE) y un grupo control (GC) que no realiza esta actividad, con mediciones pre- y postest. El ámbito incluye los pacientes ancianos diagnosticados de trastorno depresivo ingresados en la Unidad de Hospitalización Psiquiátrica de Agudos (UHPA) del Complejo Hospitalario de Navarra (España). Se plantea como estudio piloto con intención de hacerlo extensivo a otras unidades de hospitalización.

\section{Definición de la muestra}

De marzo a diciembre de 2017 se incluyeron en el estudio 64 sujetos hospitalizados en la UHPA, participantes voluntarios, que cumplían los criterios de selección. Se realizó un muestreo por conveniencia. La asig- nación a los grupos GI y GC fue aleatoria simple. La selección de individuos se realizó teniendo en cuenta los datos recogidos en la memoria de 2016 del Servicio Navarro de Salud. El número de camas es de 51, el número de ingresos, 889, y la estancia media, 19,1 días.

\section{Criterios de selección}

Los criterios de inclusión de los sujetos en la muestra fueron: edad mayor o igual a 65 años; capacidad para realizar actividades de la vida cotidiana, sin el empleo de recursos auxiliares (bastón, silla de ruedas, andador, etc.); condiciones fisiológicas que le permiten realizar el paseo o entrenamiento dirigido: colesterol total (CT: $220 \mathrm{mg} / \mathrm{dl} / \mathrm{HDL}: 40 \mathrm{mg} / \mathrm{dl}$ ), tensión arterial (TAS: $140 \mathrm{mmHg} / \mathrm{TAD}: 90 \mathrm{mmHg}$ ), índice de masa corporal (IMC 20-25), riesgo cardiovascular (CV REGICOR) que se clasifica en: bajo $(\leq 5 \%)$, moderado $(\leq 10-19 \%)$, alto $(\leq 20-39 \%)$ y muy alto $(\geq 39 \%)$ y número de fármacos prescritos ( $\mathrm{IQR})$; síntomas negativos asociados al trastorno depresivo: inhibición cognitiva y psicomotriz, tendencia al encamamiento y clinofilia y, por último, voluntad para participar y permanecer en todas las sesiones del programa de intervención.

Los criterios de exclusión fueron: personas con patologías concomitantes que puedan causar limitaciones o lesiones físicas por la realización de ejercicios físicos o que interfieran en las funciones de atención, comprensión y cognición y, también, personas que usaran medicamentos para el tratamiento de lesiones oseoarticulatorias o musculares.

\section{Variables e instrumentos de recogida de datos}

Las variables independientes son: la edad, el sexo y las condiciones fisiológicas que permiten realizar la actividad física. Las variables dependientes se agrupan en dos polos: 1) los sintomas depresivos, valorados a través de la escala de Hamilton y 2) la condición física, recogida mediante la escala de Barthel, el test de Guralnik o Short Physical Performance Battery (SPPB), medidas de movilidad en personas que son capaces de caminar por su cuenta (Get Up and Go Test) y la movilidad articular a través de la Clasificación de Resultados de Enfermería NOC (tabla 1).

El programa de intervención ED + PSECE consiste en:

1. Ejercicio dirigido (ED): dos sesiones de 90 minutos de ejercicio físico por semana, combinando: $a$ ) entrenamiento de fuerza muscular a una intensidad de trabajo entre el $40 \%$ y el 50\% de 1 RM (fuerza máxima de una repetición), entre 12 y 15 repeticiones en cada serie y un total de 2 series, con ejercicios de bíceps, tríceps, hombros, dorsal, pectoral y el músculo cuádriceps, empleando mancuernas para el trabajo de la fuerza y bandas elásticas de thera-band y $b$ ) entrenamiento de resistencia aeróbica andando en espacios interiores/exteriores, durante 20-30 minutos por sesión, a intensidades moderadas relativas de ejercicio correspondientes entre el $82 \%$ y el $100 \%$ de la frecuencia cardiaca alcanzada en el test de 6 minutos marcha. $\mathrm{Al}$ inicio de cada sesión se realizará un calentamiento con ejercicios de movilidad articular y al final de la sesión se realizarán estiramientos de los principales grupos que se han trabajo.

2. Paseos saludables en espacios curativos exteriores (PSECE): sesión semanal de paseo, con una duración mínima de 3 minutos con acompañamiento de enfermeras del equipo de investigación pertenecientes a la unidad UHPA del CHN.

El número total de sesiones de ED + PSECE mínima es de 25 (equivalente a 31,5 horas de participación activa en el programa) y el número máximo está en función de la duración media de ingreso. 
Tabla 1. Variables salud psicosocial y condición física en ancianos hospitalizados en la UHPA del CHN (España) 2017

\begin{tabular}{|c|c|}
\hline \multicolumn{2}{|r|}{ VARIABLES } \\
\hline SINTTOMAS DEPRESIVOS & CONDICIÓN FISICA \\
\hline $\begin{array}{l}\text { Humor depresivo (tristeza, desesperanza, desamparo, sentimiento } \\
\text { de inutilidad), insomnio e inhibición psicomotora (lentitud de } \\
\text { pensamiento y lenguaje, facultad de concentración disminuida, } \\
\text { disminución de la actividad motora) }\end{array}$ & $\begin{array}{l}\text { Estado dinámico de energía y vitalidad que permite a las personas llevar a cabo las } \\
\text { tareas diarias habituales, disfrutar del tiempo de ocio activo, afrontar las emergencias } \\
\text { imprevistas sin fatiga excesiva, a la vez que ayuda a evitar las enfermedades } \\
\text { hipocinéticas derivadas de la falta de actividad física }{ }^{16}\end{array}$ \\
\hline $\begin{array}{l}\text { Escala heteroaplicada, de } 17 \text { ítems, que evalúa el perfil } \\
\text { sintomatológico y la gravedad del cuadro depresivo. Proporciona } \\
\text { una puntuación global de gravedad y una puntuación en tres } \\
\text { factores o índices: melancolía, ansiedad y sueño }{ }^{18} \text {. También se ha } \\
\text { descrito un factor de vitalidad-enlentecimiento }{ }^{19}\end{array}$ & $\begin{array}{l}\text { y permite estımar cuantitativamente su grado de independencla } \\
\text { SPPB } \\
\text { Escala para valorar la capacidad funcional de las personas mayores. Consta de tres } \\
\text { pruebas: equilibrio, velocidad de la marcha y levantarse/sentarse de una silla } \\
\text { Get Up and Go Test }{ }^{0}\end{array}$ \\
\hline $\begin{array}{l}\text { Fuentes de información: } \\
\text { - Entrevista clínica } \\
\text { - Fuentes secundarias }\end{array}$ & $\begin{array}{l}\text { NOC Movilidad articular }{ }^{21} \\
\text { Clasificación de resultados de enfermería mediante criterios estandarizados de movilidad, } \\
\text { posición y equilibrio para describir y obtener resultados consecuentes a la intervención }\end{array}$ \\
\hline
\end{tabular}

CHN: Complejo Hospitalario de Navarra; UHPA: Unidad de Hospitalización Psiquiátrica de Agudos.

\section{Análisis de datos}

Los análisis estadísticos se realizan con el paquete estadístico IBMSPSS Statistics, versión 23. Los valores descriptivos se presentan como la media y la desviación típica $(\mathrm{M} \pm \mathrm{DT})$. Se realiza la prueba $t$ de Student, dado que el tamaño muestral es pequeño y su distribución es normal, utilizándose una estimación de la desviación típica en lugar del valor real. Para verificar si las desviaciones estándar de dos poblaciones normalmente distribuidas son iguales ( $\sigma^{\star}$ y Q), se emplean los estadísticos U de Mann-Whitney (prueba no paramétrica aplicada a dos muestras independientes, número de fármacos) y $\mathrm{F}$ de Fisher.

El tamańo de efecto (TE), considerado como adecuado para diseños de investigación experimentales, se valora mediante la M de McNemar, adecuada para evaluar a sujetos que participan en dos momentos diferentes del tratamiento.

\section{Aspectos éticos}

Los resultados incluidos en el presente artículo se enmarcan en un proyecto de investigación subvencionado por el Departamento de Salud del Gobierno de Navarra (Espańa) y cuenta con el informe positivo del Comité Ético de Investigación Clínica de este organismo gubernamental, ya que cumple con las normas BPC (CPMP/ICH/135/95).

\section{- RESULTADOS}

El $25 \%$ de la muestra son hombres ( $\mathrm{n}=16,8$ en GI y 8 en GC) y el $75 \%$ mujeres ( $n=48,24$ en GI y 24 en GC), no encontrándose diferencia significativa ( $\mathrm{F}$ de Fisher 0,118).

La edad media del GI es de 66,4 años y la del GC de 67,2 años. No se encuentran diferencias estadísticamente significativas con la $t$ de Student en riesgo cardiovascular medio, edad, cifras de colesterol total (HDL), tensión arterial, índice de masa corporal (IMC), riesgo cardiovascular ni en consumo medio de fármacos con la $U$ de Mann-Whitney $(p<0,05)$. La tabla 2 incluye los datos descriptivos de los 64 participantes previos al inicio de la actividad.

No se encuentran diferencias significativas entre ambos grupos en la permanencia en la Unidad con la $t$ de Student $(p 0,196)$. También se incluyen las medias de días en los que los sujetos del GI realizan actividad física del programa de intervención, tal y como se refleja en la tabla 3.

En cuanto a los resultados del análisis pre-postintervención, se observa una mejora significativa del GI en el índice de Barthel de 10 puntos $(p=0,036)$, si bien también se aprecia cierta mejoría en el grupo control. Se observa mejora de logro de autonomía en las áreas: traslado entre la silla y la cama, desplazamientos, subir y bajar escaleras, vestirse y desvestirse y aseo personal. Se aprecia una disminución en el nivel de dependencia del grupo que previamente presenta una dependencia moderada, pasando a un nivel de dependencia escasa a través de la ejercitación del programa. No se observan diferencias significativas en capacidad funcional evaluada con la escala SPPB en ninguno de los dos grupos.

El GI tiene una reducción significativa de los síntomas depresivos valorados con la escala de Hamilton de 12 puntos $(p<0,001)$; sin embargo, en el GC no se aprecia esta reducción sintomatológica $(p=0,703)$. Los ítems en los que se obtiene un cambio significativo en la puntuación son: humor depresivo, trabajo y actividades e inhibición psicomotora. El cambio se produce en nivel medio del GI, que le sitúa en depresión muy severa antes de la intervención, pasando a nivel medio de depresión moderada postintervención.

En cuanto a los resultados de enfermería, los NOC, analizando únicamente el GI se observa un ligero aumento en la puntuación media, que es significativa únicamente en el de posición corporal $(p=0,025)$, aunque está cerca de la significación el de ambulación $(p=0,054)$, lo que induce a pensar que con un tamaño de muestra mayor probablemente esto sería extensible también al de equilibrio. Concretamente, 
Tabla 2. Características descriptivas de asignación a GI/GC de ancianos hospitalizados en la UHPA del CHN (España) 2017

\begin{tabular}{|c|c|c|c|c|c|}
\hline & \multicolumn{2}{|c|}{ GI $(n=32)$} & \multicolumn{2}{|c|}{$\mathrm{GC}(n=32)$} & \multirow{2}{*}{$\begin{array}{l}\text { Valor } \\
\text { de } p\end{array}$} \\
\hline & Media & $\mathrm{DE}$ & Media & DE & \\
\hline Edad & 66,4 & 4,5 & 67,2 & 4,5 & $0,740^{\mathrm{a}}$ \\
\hline Colesterol total & 181,8 & 45,2 & 162,75 & 59,6 & $0,508^{\mathrm{a}}$ \\
\hline HDL & 47,2 & 10,1 & 35,0 & 10,1 & $0,058^{a}$ \\
\hline TAS & 137,0 & 10,8 & 135,2 & 0,9 & $0,591^{a}$ \\
\hline TAD & 85,8 & 8,6 & 84,8 & 12,6 & $0,859^{a}$ \\
\hline IMC & 29,9 & 4,4 & 32,0 & 6,2 & $0,441^{\mathrm{a}}$ \\
\hline Riesgo CV REGICOR & 7,2 & 3,0 & 10,0 & 5,6 & $0,052^{\mathrm{a}}$ \\
\hline $\begin{array}{l}\text { Número de } \\
\text { fármacos }\end{array}$ & \multicolumn{2}{|c|}{$\begin{array}{c}\mathrm{GI}(\mathrm{n}=32) \\
\text { mediana (IQR) }\end{array}$} & \multicolumn{2}{|c|}{$\begin{array}{c}\mathrm{GC}(\mathrm{n}=32) \\
\text { mediana (IOR) }\end{array}$} & $\begin{array}{l}\text { Valor } \\
\text { de } p\end{array}$ \\
\hline & 4,0 & 2,0 & 5,0 & 2,0 & $0,379^{b}$ \\
\hline
\end{tabular}

a-test.

${ }^{\mathrm{b}}$ Mann-Whitney.

CHN: Complejo Hospitalario de Navarra; CV: cardiovascular; DE: desviación estándar; GC: grupo control; Gl: grupo intervención; HDL: lipoproteínas de alta densidad; IMC: índice de masa corporal; TAD: tensión arterial diastólica; TAS: tensión arterial sistólica; UHPA: Unidad de Hospitalización Psiquiátrica de Agudos.

Tabla 3. Estancia e intervención medias en el programa de grupos Gl y GC de ancianos hospitalizados en la UHPA del CHN (España), 2017

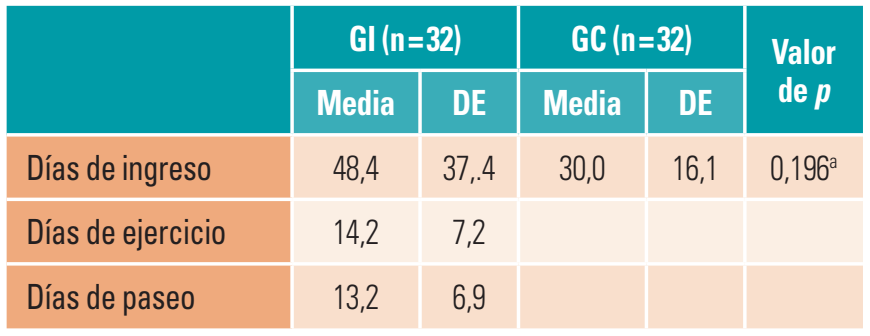

${ }^{a}$ T-test.

CHN: Complejo Hospitalario de Navarra; DE: desviación estándar; GC: grupo control; Gl: grupo intervención; UHPA: Unidad de Hospitalización Psiquiátrica de Agudos. las acciones personales para cambiar independientemente de posición corporal, con o sin dispositivo de ayuda, que mejoran significativamente son: movimientos de supinación a sedestación, de sedestación a bipedestación y flexibilidad en el tronco (doblarse por la cintura estando de pie).

En coherencia con los niveles valorados con el índice de Barthel, el porcentaje de pacientes del GI en la prueba Get Up and Go Test, asignados a la categoría normal, aumenta del pre- al post- $(33,3 \%$ vs. $58,3 \%)$ de manera significativa $(p=0,043)$ en McNemar (tabla 4).

\section{- DISCUSIÓN}

Los estudios que se ocupan de la prevalencia de la depresión en España durante los últimos 15 años evidencian que se observa un patrón de género constante, con mayor proporción de mujeres que presentan este cuadro en diferentes grupos poblacionales, siendo uno de ellos el constituido por los sujetos mayores de 65 ańos, lo que se constata en la muestra del estudio con una relación de $3 / 1^{22,23}$. Además, este grupo poblacional presenta algún grado de limitación funcional para las actividades básicas de la vida diaria (ABVD) en un 20,2\%; en nuestro estudio, la muestra que se situaba en el nivel preintervención en niveles de dependencia moderada pasa a niveles medios postintervención de dependencia escasa ${ }^{24}$.

El riesgo de tener depresión diagnosticada, con independencia de variables sociodemográficas (edad, sexo) o incluso comorbilidad con otras enfermedades, es 2,8 veces mayor en población con limitaciones funcionales para la vida diaria. Si se confirma que la limitación funcional tiene un papel importante en la depresión, también la mejora en independencia para ABVD contribuye a la disminución de la depresión, de manera concomitante, como encontramos en los resultados postintervención ${ }^{25}$. De acuerdo con los resultados obtenidos en este estudio piloto, se observa una influencia positiva del ejercicio físico en la reducción de algunos de los síntomas depresivos y en la mejoría de la aptitud funcional, lo que pretende ser un primer paso para realizar nuevas intervenciones en el ámbito de la salud mental que contemplen la actividad física acompañada como una parte más del tratamiento ${ }^{26,27}$. En este proyecto se otorga un papel primordial a la motivación para la realización de actividad física. No se requieren unas

Tabla 4. Diferencias pre-postintervención entre GI y GC de ancianos hospitalizados en la UHPA del CHN, España, 2017

\begin{tabular}{|l|c|c|c|c|c|}
\hline \multirow{2}{*}{ Barthel } & & Pre & Post & Cambio (IC95\%) & Valor de $\boldsymbol{p}^{\mathbf{2}}$ \\
\hline \multirow{2}{*}{ SPPB } & GI & $87,5(15,7)$ & $97,5(5,0)$ & $10,0(0,8,19,2)$ & 0,036 \\
\hline \multirow{2}{*}{ Hamilton } & GC & $85,0(16,8)$ & $93,5(2,9)$ & $8,5(-11,4,36,4)$ & 0,194 \\
\hline \multirow{2}{*}{ NOC Equilibrio } & GI & $9,2(2,2)$ & $10,1(2,3)$ & $0,8(-0,3,2,0)$ & 0,147 \\
\hline NOC Posición & GI & $7,2(3,0)$ & $8,0(2,9)$ & $0,8(-0,8,2,3)$ & 0,215 \\
\hline NOC Ambulación & GC & $24,2(9,3)$ & $13,1(7,3)$ & $-12,1(-17,0,-7,1)$ & $<0,001$ \\
\hline
\end{tabular}

a T-test.

CHN: Complejo Hospitalario de Navarra; GC: grupo control; GI: grupo intervención; IC: intervalo de confianza; SPPB: Short Physical Performance Battery; UHPA: Unidad de Hospitalización Psiquiátrica de Agudos. 
condiciones fisiológicas avanzadas, sino funcionalidad suficiente para participar en esta actividad, formar parte del grupo y sentirse activo/a durante el proceso de hospitalización. Este aspecto se ha tenido en cuenta a la hora de confeccionar este programa de actividad física especialmente adaptado a las necesidades de estos ancianos. Por un lado, mejora el estado de ánimo, minimizando los síntomas negativos en el plano afectivo y, por otro, la actividad motriz logrando una mejor predisposición para la realización de ejercicio físico que favorece la flexibilidad, la fuerza, el equilibrio y la coordinación. De este modo, se incide positivamente en la esfera motora y emocional ${ }^{28,29}$.

A lo largo de la intervención, los profesionales de enfermería han acompañado y supervisado la realización de un programa de ejercicio físico individual y sistemático de al menos 30 minutos diarios, con una frecuencia de tres sesiones semanales, siguiendo las recomendciones de Nystrom $^{30}$. Los cuidados de enfermería vinculados a esta actividad se dirigen a facilitar la realización del mismo, mejorar la adherencia y la predisposición, además de acompańar a los pacientes en esta actividad que promueve su bienestar físico y paralelamente puede contrarrestar los síntomas de inhibición y apatía (fig. 1). El estudio piloto da lugar a la institucionalización de la práctica terapéutica de paseo saludable durante la hospitalización, desde el Departamento de Salud del Gobierno de Navarra (España) (http://www.navarra.es/home_es/Temas/ Portal+de+la+Salud/Ciudadania/Me+cuido/Habitos+saludables/Paseos+saludables/). Esta propuesta pretende contribuir a la humanización del entorno hospitalario haciendo uso de los espacios verdes actuales del recinto hospitalario, ofreciendo evidencias de los efectos positivos del paseo terapéutico en la funcionalidad y autonomía de los pacientes y la minimización de síntomas depresivos. Es una apuesta por la incorporación de una sencilla actividad al aire libre, el paseo por los jardines, aprovechando las infraestructuras existentes y contribuyendo a la mejora de estas seńalizando varios itinerarios e incorporando otros elementos al paisaje natural. De este modo, tanto los usuarios como los familiares y/o los profesionales, así como cualquier ciudadano que lo desee, puede hacer uso de este lugar de esparcimiento, transformando un espacio na-

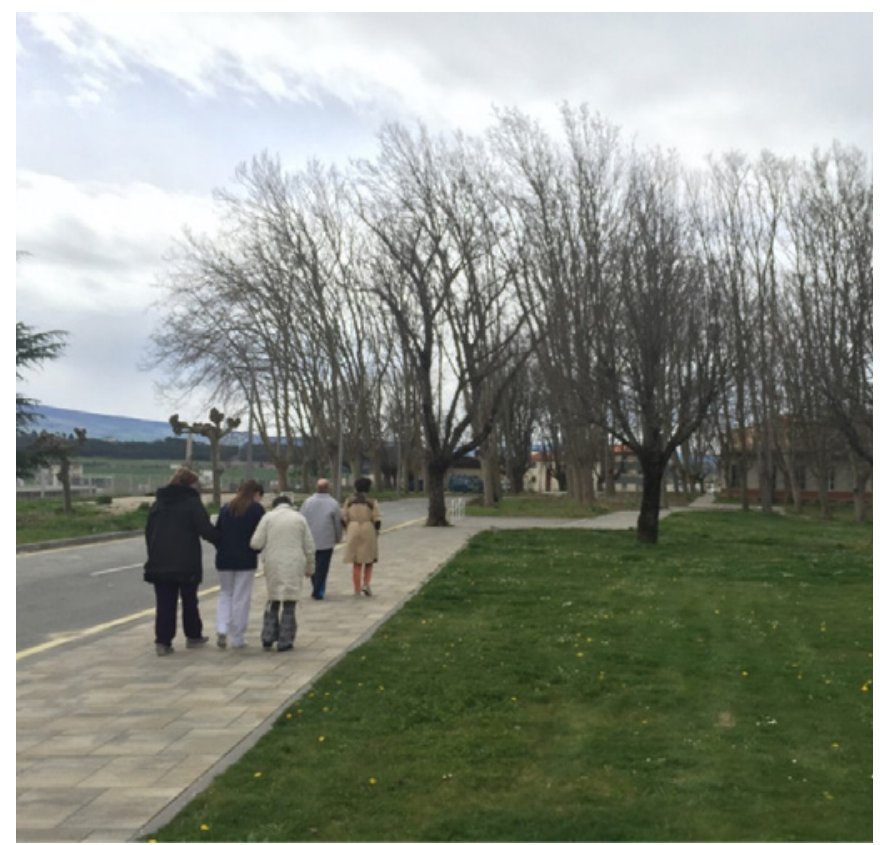

Figura 1. Paseo terapéutico acompañado por las inmediaciones del Complejo Hospitalario de Navarra. tural singular en una zona terapéutica activa para la salud y el bienestar de las personas.

En este sentido, a lo largo de este proyecto los profesionales de enfermería han acompańado y supervisado la realización de un programa de ejercicio físico individual y sistemático en el que podrían incluirse en nuevas ediciones a otros profesionales de la salud (fig. 2). Los cuidados de enfermería vinculados a esta actividad se dirigen a facilitar la realización del mismo, mejorar la adherencia y la predisposición, además de acompañar a los pacientes en esta actividad que promueve su bienestar físico y paralelamente puede contrarrestar los síntomas de inhibición y apatía. Una herramienta clave para la ejecución de este proyecto ha sido la implicación de la propia organización para apostar por nuevas estrategias que permitan incorporar la actividad física en actividades cotidianas como caminar, pasear, mirar a lo lejos en el entorno natural e intercalar tiempos para las salidas acompañados durante el ingreso en la UHP.

Consideramos que la actividad física es un elemento que mejora la salud y la calidad de las personas, cobrando una gran relevancia en la investigación referente a la promoción de la salud y prevención de la discapacidad y dependencia. Sería de gran interés realizar futuros proyectos a través de diseños cualitativos de investigación que incorporen la voz de los participantes, para conocer en primera persona la vivencia de su enfermedad, así como los condicionantes fisiológicos y psicosociales que interfieren en la motivación para realizar actividad física.

\section{- LIMITACIONES}

Al tratarse de un estudio piloto, el tamaño muestral es pequeño. La estancia media de los pacientes puede ser un factor a tener en cuenta a la hora de acompañar tanto en el ED como en el PSCE. La vulnerabilidad de los pacientes afectados por trastornos de salud mental requiere de un elevado número de profesionales. Sin embargo, la realidad actual pone de manifiesto que en los centros hospitalarios, la elevada carga de trabajo dificulta la incorporación de nuevas actividades.

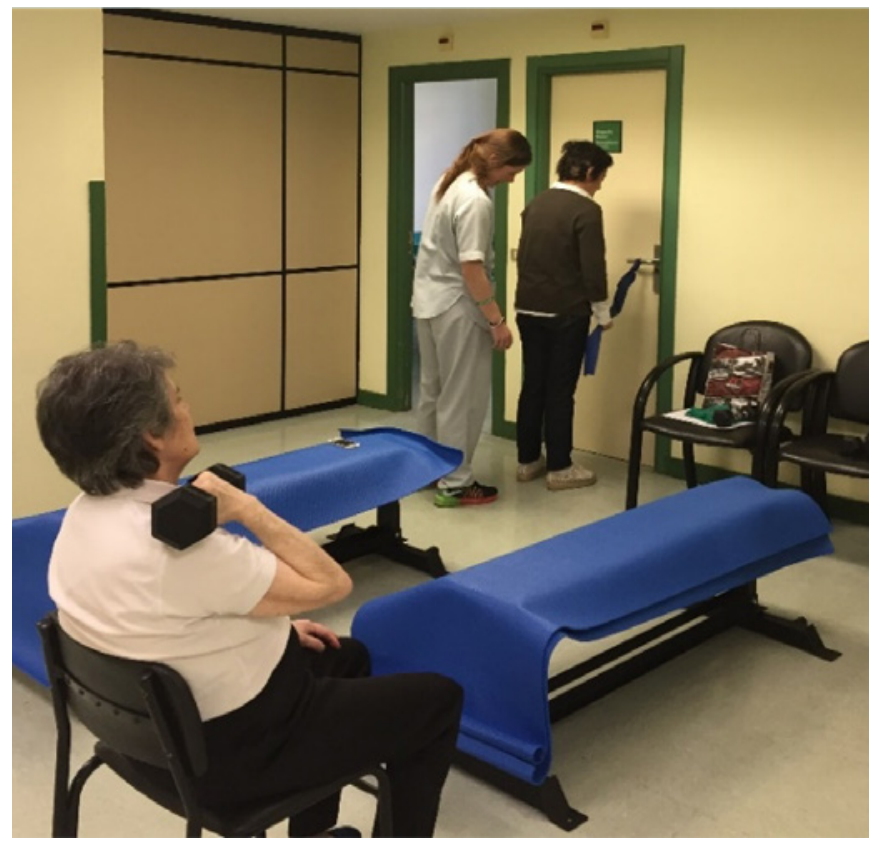

Figura 2. Actividad física grupal. Unidad de Hospitalización Psiquiátrica (2017). 


\section{- CONCLUSIÓN}

La actividad física combinada contribuye a la reducción de síntomas negativos en pacientes ancianos ingresados por trastornos depresivos, evaluados a través de la escala de Hamilton, mejora la autonomía, obetivándose un incremento de la puntuación en la escala de Barthel e incrementa la movilidad. El rol desempeñado por el profesional de enfer- mería para la adherencia al programa en una unidad de salud mental ha facilitado su ejecución e implantación en otras unidades del Complejo Hospitalario de Navarra (España)

\section{Conflicto de intereses}

Los autores declaran no tener ningún conflicto de intereses relacionado con este artículo.

\section{- BIBLIOGRAFÍA}

1. De Oliver JSP Hernández MEM, Ortega GA, Medrano LA Navarro RM. Depresión, discapacidad y sus costes. Propuestas de mejora de asistencia en Atención Primaria. Infor psicològ. 2018;1(115):5465. doi: dx.medra.org/10.14635/IPSIC.2018.115.6

2. Valdes King M, González Cáceres JA, Salisu Abdulkadir M. Prevalencia de depresión y factores de riesgo asociados a deterioro cognitivo en adultos mayores. Revista Cubana de Medicina General Integral [revista en Internet]. 2018 [citado 28 de febrero de 2019];33(4). Disponible en: http://revmgi.sld.cu/index.php/mgi/ article/view/296

3. Sendra-Gutiérrez JM, Asensio-Moreno I, Vargas-Aragón ML. Characteristics and factors associated with depression in the elderly in Spain from a gender perspective. Actas Esp Psiquiatr. [internet] 2018 [acceso 28 de febrero de 2019];45(5):185-200. Disponible en: https://europepmc.org/abstract/med/29044444. doi:10.5114/ nan.2018.81250

4. 4. Toro R, Alzate L, Santana L, Ramírez I. Afecto negativo como mediador entre intolerancia a la incertidumbre, ansiedad y depresión. Ansiedad y Estrés. 2018;24(2-3):112-8. doi.org/10.1016/j. anyes.2018.09.00

5. Schuch FB, Vancampfort D, Firth J, Rosenbaum S, Ward PB, Silva ES, et al. Physical activity and incident depression: a meta-analysis of prospective cohort studies. Am J Psychiatry. 2018;175(7):631 48. doi.org/10.1176/appi.ajp.2018.17111194

6. Sander C, Ueck P, Mergl R, Gordon G, Hegerl U, Himmerich H. Physical activity in depressed and non-depressed patients with obesity. Eating and Weight Disorders-Studies on Anorexia, Bulimia and Obesity. Eat Weigth Disord. 2018:23(2):195-203. doi: 10.1007/ s40519-016-0347-8

7. Rodríguez C, Karito E. Grado de adherencia al tratamiento antidepresivo en pacientes ambulatorios de salud mental del Hospita Nacional Edgardo Rebagliati Martins. Universidad Nacional de Trujillo. 2016. [revista en Internet]. 2016 [citado 28 de febrero de 2019]. Disponible en: http://dspace.unitru.edu.pe/handle/ UNITRU/10352

8. Akil H, Gordon J, Hen R, Javitch J, Mayberg H, McEwen B, et al. Treatment resistant depression: a multi-scale, systems biology approach. Neurosci Biobehav Rev. 2018;84:272-88. doi.org/10.1016/jneubiorev.2017.08.019

9. Cohen ZD, DeRubeis RJ. Treatment selection in depression. Annu Rev Clin Psycho. 2016:14:209-36. doi: 10.1146/annurev-clinpsy-050817-084746

10. Kaseva K, Dobewall H, Yang X, Pulkki-Råback L, Lipsanen J, Hintsa, et al. Physical Activity, Sleep, and Symptoms of Depression in Adults-Testing for Mediation. Med Sci Sports Exerc. 2019. doi: 10.1249/MSS.0000000000001896

11. Choi KW, Chen CY,Stein MB, Klimentidis YC, Wang MJ, Koenen $\mathrm{KC}$, et al. Assessment of bidirectional relationships between physical activity and depression among adults: a 2-sample mendelian randomization study. JAMA Psychiatry. 2019:1;76(4):399-408. doi:10.1001/jamapsychiatry.2018.4175

12. Organizacion Mundial de la salud. $66{ }^{\text {a }}$ Asamblea Mundial de la Salud. Proyecto de plan de acción integral sobre salud menta 2013-2020. Informe de la Secretaría. No. A66/10 Rev. 1. 2013 [citado 28 de febrero de 2019]. Disponible en: https://apps.who.int/ iris/bitstream/handle/10665/150914/A66_10Rev1-sp.pdf

13. Melissa de Freitas L, Talita Portela C, Lyliam Midori S, Mendes DV, Barbosa de PB, de Fátima LA. Incidência de quedas e ações preventivas em um Hospital Universitário. Rev. esc. enferm. USP [Internet]. 2018 [citado 28 de febrero de 2019];52:e03308. Disponible en: http://www.scielo.br/scielo.php?script=sci_arttext\&pi$d=$ S0080-62342018000100405\&lng=en. Epub Apr 12, 2018. http:// dx.doi.org/10.1590/s1980-220x2017024203308.

14. García BP, González SM, Muñoz AMC, Antón-Solanas I, CabaIlero VG, Vela RJ. Uso de medicamentos asociados al riesgo de caídas en ancianos no institucionalizados. Rev. esc. enferm. USP [Internet]. 2018 [citado 28 de febrero de 2019];52:e03319. Disponible en: http://www.scielo.br/scielo.php?script=sci_arttext\&pi$d=$ S0080-62342018000100407\&lng=en. Epub Apr 16, 2018. http:// dx.doi.org/10.1590/s1980-220x2017012603319.

15. Raatikainen I, Vanhala M, Mäntyselkä P, Heinonen A, Koponen H, Kautiainen $\mathrm{H}$, et al. Does level of leisure time physical activity, in a sample of patients with depression, predict health care utilization over a subsequent 5-year. Mental Health and Physical Activity. 2018: 15:40-44. doi.org/10.1016/..mhpa.2018.06.007

16. Bouchard C, Shephard RJ. Physical activity, fitness, and health: The model and key concepts. En: Bouchard C, Shephard RJ, Stephens T, eds. Physical activity, fitness, and health: International proceedings and consensus statement. 1994.

17. Ramos-Brieva, JA. Validación de la versión castellana de la escala de Hamilton para la depresión. Actas Luso Esp Neurol Psiquiatr. 1986;14:324-34. Disponible en: http://ci.nii.ac.jp/ naid/10017358497/en/ doi: 10.1016/S0025-7753(02)72429-9

18. Dyer K, Hooke G, Page AC. Development and psychometrics of the five item daily index in a psychiatric sample. J Affect Disord. 2014:152(1):409-15. doi: 10.1016/i.jad.2013.10.003

19. Tollefson GD, Rampey AH, Enas GG, Potvin JH. Does pharmacotherapy induce paradoxical worsening in some patients? Depression.
1993:1(2):105-7. Disponible en: http://doi.wiley.com/10.1002/depr. 3050010207

20. Mathias S, Nayak US, Isaacs B. Balance in elderly patients: the "get-up and go" test. Arch Phys Med Rehabil. 1986;67(6):387-9. Disponible en: https://www.ncbi.nlm.nih.gov/pubmed/3487300

21. Moorhead S, Johnson M. Clasificación de resultados de enfermería (NOC). Elsevier Health Sciences. 2009. ISBN: 8480868414.

22. Fernández FC, Fernández FC, Martínez ÁM, Martín ABB, Pérez-Fuentes $\mathrm{M}$ del $\mathrm{C}$, Jurado $\mathrm{M}$ del MM, et al. Prevalencia de la depresión en España: Análisis de los últimos 15 años. Eur J Investig Heal Psychol Educ [Internet]. 2015:5(2):267-79. https://doi. org/10.20320/rfcsudes.v2i1.249 26

23. Seney ML, Huo Z, Cahill K, French L, Puralewski R, Zhang J, et al. Opposite molecular signatures of depression in men and women. Biolog Psych. 2018;84(1):18-27. doi: 10.1016/j biopsych.2018.01.017

24. Álvarez-Moreno B, Aguilar-Palacio I. Limitación funcional y depresión en la población anciana española [Internet]. 2017. Disponible en: http://zaguan.unizar.es/record/65564

25. Cadore EL, Rodríguez-Mañas L, Sinclair A, Izquierdo M. Effects of Different Exercise Interventions on Risk of Falls, Gait Ability, and Balance in Physically Frail Older Adults: A Systematic Review. Rejuvenation Res. 2013;16(2):105-14. doi: 10.1089/rej.2012.1397

26. Knapen J, Vancampfort $D$, Moriën $Y$, Marchal $Y$. Exercise therapy improves both mental and physical health in patients with major depression. Disability and rehab. 2015;37(16):1490-5. doi: 10.3109/09638288.2014.972579

27. Stubbs B, Vancampfort D, Rosenbaum S, Ward PB, Richards J Soundy $A$, et al. Dropout from exercise randomized controlled trials among people with depression: a meta-analysis and meta regression. J Affect Disord. 2016;190:457-66. doi: 10.1016/j. jad.2015.10.019

28. Koohsari MJ, Badland H, Mavoa S, Villanueva K, Francis J, Hooper $P$, et al. Are public open space attributes associated with walking and depression?. Cities. 2018;74:119-25. https://doi.org/10.1016/j.cities.2018.09.020

29. Sancassiani F, Machado S, Preti A. Physical activity, exercise and sport programs as effective therapeutic tools in psychosocial rehabilitation. Clinical practice and epidemiology in mental health. CP \& EMH. 2018;14:2-6. doi: 10.1016/j.revinf.2013.11.010

30. Nyström MB, Neely G, Hassmen P, Carlbring P. Treating major depression with physical activity: a systematic overview with recommendations. CBT. 2015;44(4):341-52. doi: 10.1016/i. jad.2017.03.018 